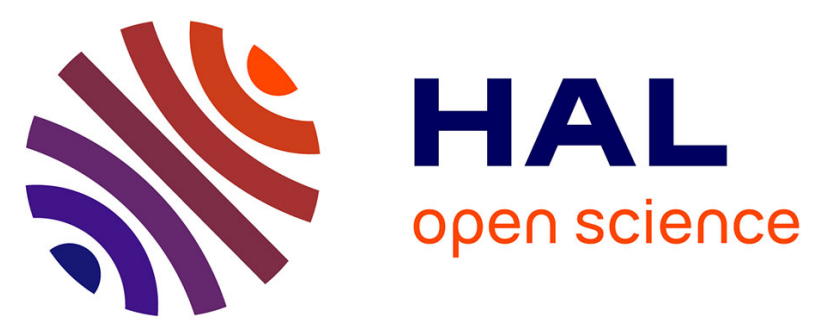

\title{
Improvements of global models for the determination of the kinetic parameters associated to the thermal degradation of lignocellulosic materials under low heating rates
}

Alain Brillard, Jean-Francois Brilhac

\section{To cite this version:}

Alain Brillard, Jean-Francois Brilhac. Improvements of global models for the determination of the kinetic parameters associated to the thermal degradation of lignocellulosic materials under low heating rates. Renewable Energy, 2019, 146, pp.1498-1509. 10.1016/j.renene.2019.07.040 . hal-03280002

\section{HAL Id: hal-03280002 \\ https://hal.science/hal-03280002}

Submitted on 25 Oct 2021

HAL is a multi-disciplinary open access archive for the deposit and dissemination of scientific research documents, whether they are published or not. The documents may come from teaching and research institutions in France or abroad, or from public or private research centers.
L'archive ouverte pluridisciplinaire HAL, est destinée au dépôt et à la diffusion de documents scientifiques de niveau recherche, publiés ou non, émanant des établissements d'enseignement et de recherche français ou étrangers, des laboratoires publics ou privés.

\section{(c) (1) $\$$}

Distributed under a Creative Commons Attribution - NonCommerciall 4.0 International 
1. Improvements of global models for the determination

A. Brillard*, J.F. Brilhac

Université de Haute-Alsace, Laboratory Gestion des Risques et Management, 3bis rue Alfred Werner, 68093 Mulhouse Cedex, France

\begin{abstract}
The study presents improvements of global models, among which the Extended Independent Parallel Reaction (EIPR) model, which can be used for the determination of the kinetic parameters associated to the pyrolysis of a lignocellulosic material under low temperature ramps. The number of constituents of the lignocellulosic material and their proportions to be involved in the simulations are linked to characterizations of the material. A quartic objective function to be minimized is proposed to simulate in a more efficient way the slow pyrolysis of the material and which leads to a unique set of optimal values of the kinetic parameters. Three different biomass are considered in the present study as examples: a T-shirt sample, Cameroonian palm nut fibers and Russian hydrolysis lignin. The good agreement between the experimental and simulated mass and mass rate curves validates the values of the kinetic parameters thus determined. These values are finally compared to that obtained when applying the differential isoconversional method (DIC) or the Distributed Activation Energy Model (DAEM).

Key words:

Biomass pyrolysis

Biomass characterizations

Kinetic modeling

Determination of kinetic parameters

Extended Independent Parallel Reaction model
\end{abstract}


* Corresponding author.

E-mail address: alain.brillard@uha.fr (Alain Brillard)

\section{Introduction}

The slow pyrolysis of biomass allows to produce bio-oils, biochar, or chemicals, see for example [1], the review in [2] and the references therein. Torrefaction is another situation where a slow pyrolysis process is performed up to temperatures generally less than $400{ }^{\circ} \mathrm{C}$, to produce an hydrophobic material whose chemical and physical properties differ from that of the original material, [3].

During pyrolysis, mechanical and chemical degradations occur in the material, the long chain polymers being broken, [4]. Modeling the slow pyrolysis of different materials has been the subject of many researches for now many years. Global models or methods start from the same first-order ordinary differential equation which describes the evolution of the mass of the material, or of the associated extent of conversion, with respect to time. This equation includes a reaction function to be chosen and kinetic parameters (preexponential factors and activation energies) to be determined. Considering a lignocellulosic representation of the material, such an ordinary differential equation may be associated to the thermal degradation of each constituent of the material. The reaction function, the number of constituents and their proportions in the material have to be determined. The optimal values of the kinetic parameters may result either from the direct resolution of the differential equation using a numerical software or from its resolution after the introduction of approximations. Performing such a kinetic analysis for a given material, in complement to the classical proximate and ultimate analyses and other characterizations, allows to compare its kinetic parameters to that of other materials submitted to the same experimental conditions.

The Extended Independent Parallel Reaction model has been developed to determine the evolution of the mass of each constituent of a material with respect to time, assuming that these constituents are being degraded in an almost independent way, [5], [6]. For each constituent of the material, the EIPR model returns a unique set of two kinetic parameters (pre-exponential factor and activation energy) through the direct resolution of the differential equation which simulates the mass loss of this constituent versus time. Other methods or models consider the material as global. For example, the differential isoconversional method considers a unique differential equation 
for the material. But instead of solving this equation, this method derives values of the kinetic parameters applying the logarithm to the two members of the differential equation, taking into account at least three different temperature ramps and plotting regression lines for successive values of the extent of conversion, see [7] for a general presentation of the isoconversional methods with recommendations on their application. The kinetic parameters are here global for the material but their values depend on that of the extent of conversion hence on the time parameter. Model-free methods such as Ozawa-Flynn-Wall, Friedman and Kissinger-Akahira-Sunose intend to remove the presence of the reaction function following quite the same procedure as the isoconversional methods, [9] and [10] among other works. The DAEM method considers an infinite series of parallel reactions which occur during the thermal degradation of the material. These first-order differential equations are weighted with probability density functions.

Other models, among which Computational Fluid Dynamics (CFD), have been introduced which intend to describe the behavior of a particle in a reactor on the basis of balance or conservation equations, see [11] and [12], for example. In [13], the DAEM model has been coupled to CFD simulations. In [14], CFD modeling has been applied to investigate the particle shrinkage and the intra-particle heat conduction along biomass fast pyrolysis. In [15], the authors discuss the major trends and roadblocks with respect to CFDaided process intensification of biomass pyrolysis. Such numerical methods can be qualified as local ones, as they consider different levels in the bed. They are especially useful in the case where diffusional limits or transport phenomena may occur during the thermal degradation process, for example during fast pyrolysis processes or in reactors which contain higher amounts of materials than in a thermobalance. In the experimental background of the present study such diffusional limits and transport phenomena are certainly reduced.

In the present study, the Extended Independent Parallel Reaction model is the main tool which is applied to simulate the pyrolysis of three different biomass under the same low temperature ramp of $10{ }^{\circ} \mathrm{C} / \mathrm{min}$. Biomass are lignocellulosic materials as they are composed of extractives, hemicellulose, cellulose and lignin (apart from moisture, char and ash), whose proportions and intricate structures vary from one material to another one. For each biomass, the number of constituents and their proportions to be considered are derived taking into account both the characterizations of this biomass, and especially that obtained when applying Van Soest's protocol [16], and 
the mass rate curve obtained from thermogravimetric analyses. To determine the optimal kinetic parameters, the choice of an objective function to be minimized is then discussed. A quartic objective function is here proposed which leads to a unique set of optimal kinetic parameters. The uniqueness of the optimal set of kinetic parameters a priori prevents from the occurrence of a possible kinetic compensation effect as observed in such situations, see the recent paper [17], for example. Once the kinetic parameters have been returned by the global model, it is possible to solve the set of differential equations which describe the evolution with respect to the time parameter of the mass of each constituent of the material. This leads to simulated mass and mass rate curves. The maximal difference between the experimental and simulated mass rate curves is computed which validates the simulations in the present context. The results of the simulations of the pyrolysis process returned by the EIPR model are finally compared to that returned by the differential isoconversional method or by the Distributed Activation Energy Model, see also [8] for a comparison of the values of the kinetic parameters associated to a biomass pyrolysis obtained through different models.

\section{Methods}

\subsection{Materials and their characterizations}

Three biomass are considered in the present study: commercial T-shirt cotton samples, Cameroonian palm nut fibers and Russian hydrolysis lignin. Palm nut fibers are by-products of palm oil production. They are obtained after extraction of palm oil, just separating them from the nuts. Hydrolysis lignin is a by-product of the technological cycles concerning the production of fuels and chemicals, through either a percolation (Scholler process) or a low-temperature (Bergius process) hydrolysis of wood, [18]. These three materials may be considered as renewable sources for energy production. In France, the amount of used cotton fabrics is estimated at 700,000 tons/year, www.ecotextile.tm.fr/ecotextile-metier.html. Cameroon was the 16th producer of palm oil in the world in 2013 with 225,000 tons. Wood hydrolysis plants were mainly built in the former USSR, Bulgaria, China and Korea. All these plants produced substantial amounts of hydrolysis lignin, at most 1.5 million tons each year.

Because some experiments require a constant granulometry, the T-shirt pieces have been cut in squares of $0.5 \mathrm{~cm}^{2}$. Palm nut fibers and hydrolyzed lignin have been milled and sieved to the particle range $0.5-1 \mathrm{~mm}$. 
Different experiments have been performed to characterize these materials: proximate and ultimate analyses, measures of the higher heating value and determination of the proportions of extractives, hemicellulose, cellulose and lignin. Each experiment has been realized at least three times to reduce possible deviations.

\subsubsection{Proximate and ultimate analyses}

The results of the proximate analyses performed on the three biomass under consideration are gathered in Table 1.

\section{Table 1}

Proximate analyses of the three biomass (daf $=$ dry ash free, ar $=$ as received, $\mathrm{db}=$ dry basis).

\begin{tabular}{lcrrrrr}
\hline & $\begin{array}{c}\text { Volatile } \\
\text { wt\%, daf }\end{array}$ & $\begin{array}{c}\text { Moisture } \\
\text { wt\%, ar }\end{array}$ & $\begin{array}{c}\text { Ash } \\
\%, \mathrm{db}\end{array}$ & \multicolumn{2}{c}{ HHV } & LHV $/ \mathrm{kg}$ \\
\hline T-shirt & 89.9 & 10.1 & 6.0 & 0.1 & 16.4 & 13.9 \\
Palm fiber (ar) & 84.2 & 15.8 & 16.0 & 7.4 & 22.6 & 22.0 \\
Hydrol. lignin & 69.0 & 31.0 & 5.5 & 4.5 & 19.5 & 18.6 \\
\hline
\end{tabular}

The moisture content of each material has been measured putting a sample in a porcelain pan which is then placed in an oven and heated up to $105{ }^{\circ} \mathrm{C}$ during 45 minutes. Then the sample was stored in a desiccator during a cooling process and weighed. The process was repeated until the sample mass remains almost constant. A Nabertherm muffle furnace has been used to determine the ash content at $550{ }^{\circ} \mathrm{C}$, according to XP CEN / TS 14775. The higher heating value (HHV) has been determined placing the sample in a metal crucible (accuracy $0.1 \mathrm{mg}$ ) in a IKA C200 calorimeter. The lower heating value (LHV) has been deduced from the HHV, removing the energy required to bring the moisture content outside the sample, according to the formula:

$$
L H V=H H V-L_{v}\left(\frac{M}{100}+\frac{M_{\mathrm{H}_{2} \mathrm{O}} H}{200 M_{H}}\right),
$$

where $L_{v}=2486 \mathrm{~kJ} / \mathrm{kg}$ is the latent heat of water vaporization at $273 \mathrm{~K}, M$ is the moisture content (\%), $M_{\mathrm{H}_{2} \mathrm{O}}=18 \mathrm{~g} / \mathrm{mol}$ is the molar mass of water, $M_{\mathrm{H}_{2} \mathrm{O}}=1 \mathrm{~g} / \mathrm{mol}$ is the molar mass of hydrogen and $H$ is the percentage of hydrogen of the sample and given in Table 2 below. 
Palm fiber has the greater percentages of moisture and ash among the three materials. It has also the greater higher and lower heating values. Tshirt has the higher volatile percentage, the lower fixed carbon percentage and the lower higher and lower heating values. Hydrolysis lignin has the higher fixed carbon percentage.

The results of the ultimate analyses performed on the three materials under consideration are gathered in Table 2.

\section{Table 2}

Ultimate analyses of the three materials.

\begin{tabular}{lccccr}
\hline & C & H & O & N & \multicolumn{1}{c}{ S } \\
\hline T-shirt & 45.5 & 6.6 & 47.5 & 0.3 & $<0.1$ \\
Palm fiber & 62.0 & 9.1 & 27.4 & 1.3 & 0.2 \\
Hydrol. Lignin & 59.5 & 6.6 & 33.7 & 0.2 & $<0.1$ \\
\hline
\end{tabular}

Palm fiber has the higher percentage of carbon among the three materials. T-shirt has the higher oxygen percentage. In each material, the sulfur percentage is very low. This is interesting when considering these materials for energy production, as sulfur is known to degrade the devices.

\subsubsection{Van Soest's protocol}

In [16], Van Soest et al. proposed a protocol to determine the fractions of extractives, hemicellulose, cellulose and lignin in a lignocellulosic material. It consists of four stages. In the first stage, a neutral detergent fiber is used to solve the extractive part of the sample. In the second stage, an acid detergent fiber is used to solve the hemicellulose part of the sample. In the third stage, the cellulose part is removed using sulfuric acid at $72 \%$ in mass. In the final fourth stage, the lignin part is removed through a calcination process. Between each stage, the sample is weighed. The chemical composition of these four constituents may be summarized as follows, [19], [2], [20], [4]:

- The extractives mainly include waxes, fats, resins, tannins, sugars, starches, pigments... The maximal mass rate for extractives is approximately reached at $205^{\circ} \mathrm{C}$.

- Hemicellulose is composed of short-chain heteropolysaccharides and presents anamorphous and branched structure. Although the shape of the polysaccharide chain is similar to that of cellulose, the degree 
of polymerization of hemicellulose is only approximately 200 on average, [21]. The monosaccharide units constituting hemicellulose include mainly hexoses (glucose, mannose and galactose) and pentoses (xylose and arabinose), as well as some other low-content saccharides (rhamnose and fructose). In addition, there are some uronic acids (4-Omethyl-d-glucuronic acid, d-glucuronic acid, and d-galacturonic acid) and acetyl groups in the hemicellulose structure. The maximal mass rate for hemicellulose is approximately reached at $270{ }^{\circ} \mathrm{C}$.

- A cellulose molecule is a long-chain, linear polymer $\left(\mathrm{C}_{6} \mathrm{H}_{10} \mathrm{O}_{5}\right)_{n}$, made up of 7000 to 12,000 D-glucose monomers. Individual cellulose molecules organize to form bundles, which are associated into larger parallel fiber structures. In the first stages of its pyrolysis process, cellulose is being depolymerized in order to build active cellulose. Then follow a solid phase and a liquid phase. At around $260{ }^{\circ} \mathrm{C}$, the liquid phase reaches the ebullition point and interacts with the solid phase. At temperatures higher than $230{ }^{\circ} \mathrm{C}$, the main constituents emitted by cellulose are: hydroxyacetaldehyde, glyoxal, methanol, water, 5-hydroxymethyl-furfural, carbon monoxide and carbon dioxide. The thermal degradation of cellulose is essentially due to the scission of polymeric chains, a number of $\beta$-glucosidic bonds being broken [22], [23]. The cellulose degradation process can be characterized in terms of the degree of polymerization with respect to the time parameter, leading to the kinetic equations derived from the first or pseudo-zero order Ekenstam's relationship, [24], for example. The maximal mass rate for cellulose is approximately reached at $327^{\circ} \mathrm{C}$.

- Lignin is mainly anamorphous tridimensional polymer composed of three basic units: p-coumaryl (4-hydroxycin-namyl), coniferyl (3-methoxy4hydroxycinnamyl) and sinapyl (3,5-dimethoxy4-hydroxycinnamyl) alcohols. The thermal degradation of lignin usually occurs in a wide temperature range.

The chemical reactions which occur during the pyrolysis of a lignocellulosic material have been described in [25].

The values of the mass fractions of extractives, hemicellulose, cellulose and lignin are gathered in Table 3, with a sum equal to $100 \%$ for each material. 
Table 3

Mass fractions (\%) of the four constituents for the three biomass, as determined through Van Soest's protocol.

\begin{tabular}{lrrrr}
\hline & Extractives & Hemicellulose & Cellulose & Lignin \\
\hline T-shirt & 7.2 & 0.8 & 91.2 & 0.8 \\
Palm fiber & 47.4 & 16.6 & 23.3 & 12.6 \\
Hydrol. Lignin & 12.2 & 2.3 & 19.2 & 66.3 \\
\hline
\end{tabular}

The four samples here present large differences. Palm fibers contain a very large proportion (almost half) of extractives. On the contrary, hydrolysis lignin contains low proportions of extractives and hemicellulose, but a very high proportion $(2 / 3)$ of lignin. T-shirt sample contains a very high proportion of cellulose. Cotton is indeed known to have a very high cellulose percentage. These proportions will impact the thermal degradation profile of the materials as exposed in Figure 1 in section 3.1.

\subsubsection{Thermogravimetric analysis under nitrogen}

Once these materials have been characterized, their thermal degradation profiles have been analyzed performing thermogravimetric analyses in a thermobalance (Q600, TA Instruments) under a non-oxidative (pure nitrogen) atmosphere. A sample of around $10 \mathrm{mg}$ was put in the alumina crucible of the thermobalance and heated from ambient temperature to $900{ }^{\circ} \mathrm{C}$, under a low temperature rate $\left(10{ }^{\circ} \mathrm{C} / \mathrm{min}\right)$. The gas flow rate was fixed at $60 \mathrm{~mL} / \mathrm{min}$. Reproducibility tests (at least three) were performed for each biomass, with good agreement.

\subsection{Modeling the thermal degradation of materials through the EIPR model}

Different methods and models have been proposed to simulate the thermal degradation of materials under low temperature ramps and under a non-oxidative atmosphere. The present study will focus on the EIPR model, following [5], [6] and [26]. The EIPR model superimposes the thermal degradations of the initial masses of the constituents of the sample, these degradations being supposed to occur in an almost independent way. The initial mass $m_{\text {ini }}$ of the sample is decomposed as $m_{\text {ini }}=m(0)+m_{\text {ash }}+m_{\text {hum }}$, where $m(0)$ (resp. $m_{\text {ash }}, m_{\text {hum }}$ ) is the mass of volatiles which are emitted and of char which is produced (resp. ashes, humidity) in the sample. At time $t$, the 
remaining mass $m(t)$ of the sample which can produce volatiles and char is given by:

$$
m(t)=\sum_{i=1}^{I} m_{i}(t)=\sum_{i=1}^{I}\left(m_{i}(0)-m_{v o l, i}^{e}(t)\right),
$$

where $m_{i}(t)$ is the mass of volatiles and of char contained in the constituent $i(i=1, \ldots, I)$ of the sample at time $t, m_{i}(0)$ is the initial mass of the constituent $i$, which may be computed as a fraction of the overall mass of the sample: $m_{i}(0)=c_{i} m(0)$ and $m_{v o l, i}^{e}(t)$ is the mass of volatiles emitted by the constituent $i$ of the sample $(i=1, \ldots, I)$, at time $t$. The number of constituents of the material and the fraction coefficients $c_{i}$ of these different constituents have to be determined, which is not an easy task. The overall number $I$ of constituents of the lignocellulosic material to be considered in the EIPR model is determined both taking into account the mass fractions of the constituents as indicated in Table 3 and observing the number of peaks of the mass rate curve. The fraction coefficients of these constituents may be deduced from the proportions of extractives, hemicellulose, cellulose and lignin given in Table 3.

The evolution of the mass of volatiles emitted by the constituent $i$ is written as:

$$
\left\{\begin{array}{l}
\frac{d m_{v o l, i}^{e}}{d t}(t)=k_{i}(T(t)) f\left(m_{v o l, i}^{e}(t)\right), \\
m_{v o l, i}^{e}(0)=0,
\end{array}\right.
$$

where $T(t)$ is the temperature at time $t$ in the sample which is supposed to be homogeneous inside the whole sample (expressed in $\mathrm{K}$ ). Here $f$ is a reaction function, which may be expressed in terms of the overall extent of conversion $\alpha$ defined at time $t$ through:

$$
\alpha(t)=\frac{m_{0}-m(t)}{m_{0}-m_{f}},
$$

where $m_{0}$ is the initial mass, $m(t)$ is the mass at time $t$ and $m_{f}$ is the final mass of the sample, through:

- the first-order, or Mampel, reaction function: $f(\alpha)=1-\alpha$, 
- then Avrami-Erofeev reaction function of second-order:

$$
f(\alpha)=2(1-\alpha)(-\ln (1-\alpha))^{1 / 2},
$$

- the Prout-Tompkins reaction function: $f(\alpha)=\alpha(1-\alpha)$,

- the chain scission function $f(\alpha)=2\left(\alpha^{0.5}-\alpha\right)$,

- the 3D diffusion function:

$$
f(\alpha)=\frac{1.5(1-\alpha)^{1 / 4}}{1-(1-\alpha)^{1 / 3}},
$$

among many others. In most cases, the first-order reaction function gives satisfying results. The Avrami-Erofeev reaction function of second-order has been successfully used to simulate the pyrolysis of cotton make-up remover in $[28]$.

In the above equation $(1)_{1}$, the kinetic constant $k_{i}(T)$ obeys an Arrhenius law: $k_{i}(T)=A_{i} \exp \left(-E a_{i} / R T\right)$, where $A_{i}$ (resp. $\left.E a_{i}\right)$ is the pre-exponential factor (resp. the activation energy) for the devolatilization stage of the constituent $i$.

The problem (1) is solved using the numerical software Scilab (version 6.0.1) and especially its routines 'ode' and 'datafit', to determine the optimal set of kinetic parameters $\left(A_{i}, E a_{i}\right)$. For this optimization process, an objective function (error) is built $F\left(\left(\frac{d m}{d t}\right)_{\exp },\left(\frac{d m}{d t}\right)_{s i m}\right)$, where $\left(\frac{d m}{d t}\right)_{s i m}=$ $\sum_{i=1, \ldots, I} \frac{d m_{v o l, i}^{e}}{d t}$ is the simulated mass rate and $\left(\frac{d m}{d t}\right)_{\exp }$ is the experimental mass rate. The objective function should lead to a unique set of kinetic parameters, for example to prevent from a possible kinetic compensation effect. Once the objective function $F$ has been chosen and choosing initial values of the kinetic parameters, the Scilab routine datafit finds the optimal values of the kinetic parameters which minimize this objective function. Further observe that the numerical resolution of the problem (1) with the Scilab routine ode leads to almost negligible errors.

\section{Results and discussions}

\subsection{Thermal degradations under nitrogen}

The mass loss and mass rate curves describing the thermal degradation of each sample under nitrogen and under a temperature ramp of $10{ }^{\circ} \mathrm{C} / \mathrm{min}$ are presented in Fig. 1. 


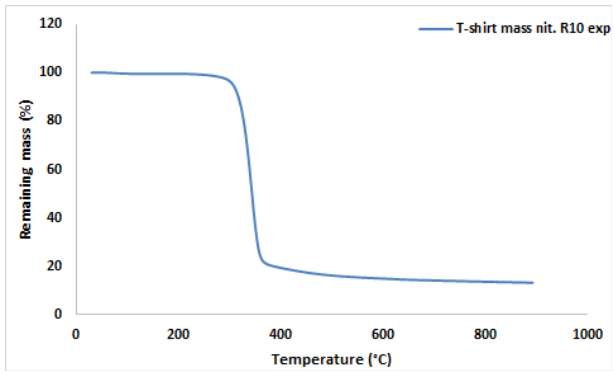

a)

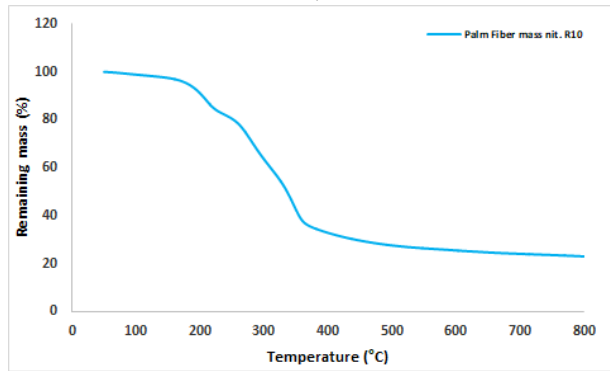

c)

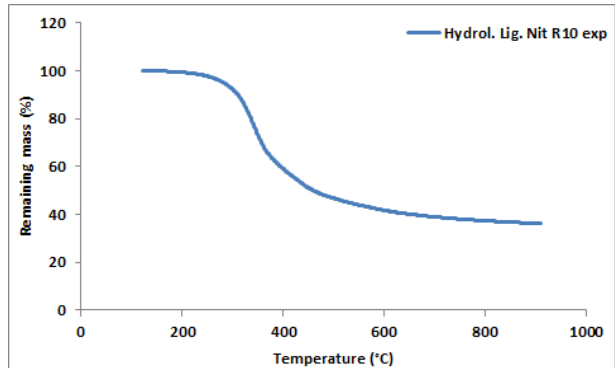

e)

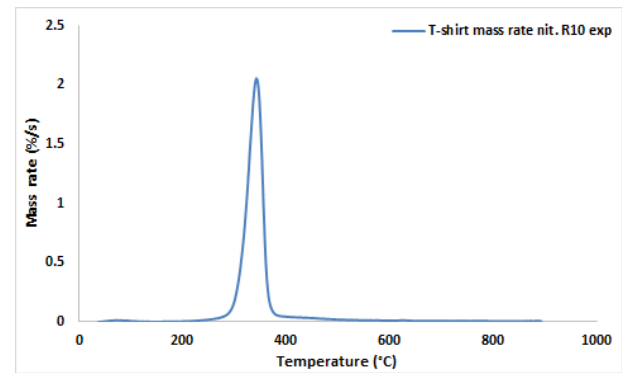

b)

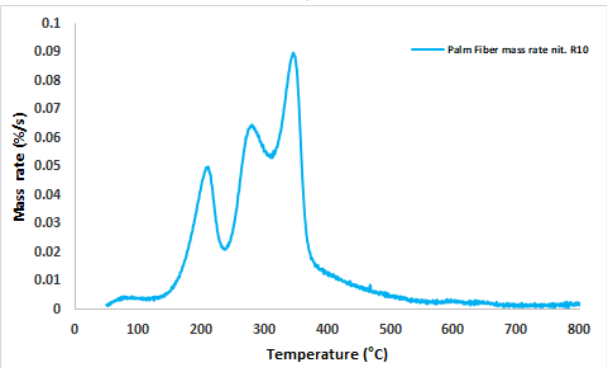

d)

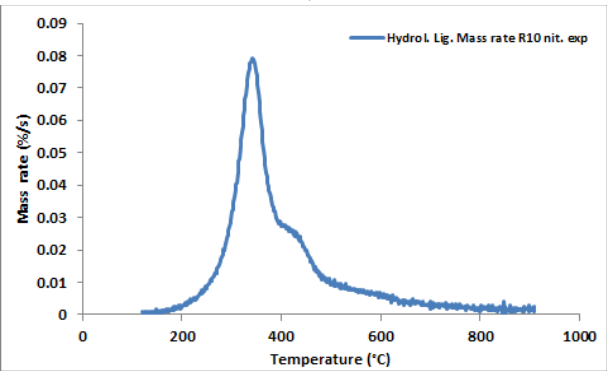

f)

Fig. 1. Mass loss and mass rate curves for T-shirt $(a)$ and $b)$ ), palm fiber $(c)$ and $d)$ ) and hydrolysis lignin $(e)$ and $f)$ ) under nitrogen and a temperature ramp of $10{ }^{\circ} \mathrm{C} / \mathrm{min}$.

Due to the high presence of cellulose in the T-shirt sample, a high and thin devolatilization peak appears whose maximal height occurs at around $344{ }^{\circ} \mathrm{C}$. Another small peak appears before $120{ }^{\circ} \mathrm{C}$ which corresponds to the moisture evaporation. After the main peak, a small tail appears which ends at almost $500{ }^{\circ} \mathrm{C}$. 
Surprisingly, the mass loss rate curve of palm fibers exhibits four peaks. The first one occurs before $120{ }^{\circ} \mathrm{C}$ and surely corresponds to moisture evaporation. The three other ones which respectively occur at 211,281 and $346^{\circ} \mathrm{C}$ correspond to devolatilization stages of the different constituents of the material, among which are the extractives whose proportion is very high, see Table 3. Notice that the maximal height of the peaks increases with the temperature, which gives an idea of the relative mass fraction of each constituent to be considered when modeling the thermal degradation of the palm fibers through the EIPR model. At the right-hand side of the fourth peak, a quite important tail appears which corresponds to the end of the lignin decomposition.

The mass rate of hydrolysis lignin presents a unique devolatilization peak whose maximal height occurs at around $341^{\circ} \mathrm{C}$. But an important shoulder appears at the right-hand side of this unique peak and a long tail ends at around $800{ }^{\circ} \mathrm{C}$.

The three materials present very different thermogravimetric profiles under pyrolysis, which justifies their choice, apart from their different origins, characterizations as indicated in the previous section and uses as renewable combustible.

Because the moisture evaporation stage is not the core of the present study, the simulations of the thermal degradations of these materials will be presented in the temperature range $150-850{ }^{\circ} \mathrm{C}$.

\subsection{Determination of the kinetic parameters associated to the pyrolysis process}

In the present study, the above problem (1) is solved first considering a number of constituents depending on the material. In the simple case of the T-shirt sample, a unique constituent may be considered which corresponds to cellulose. This is confirmed looking at the proportions of the different constituents given in Table 3. In the case of palm fibers, four constituents have to be considered which correspond to extractives, hemicellulose, cellulose and lignin. But comparing the proportions given in Table 3 and the mass rate curve of palm fiber under nitrogen (Figure $1 d)$ ), these proportions are not really adapted. From the area located under each peak or shoulder, it is indeed possible to get an estimate of the proportion of each constituent which is being removed. In the case of palm fibers, this means that part of the extractives are being emitted with the other three constituents. Modeling the pyrolysis of palm fibers with the proportions of Table 3 in fact leads to a poor agreement with the experimental results, whatever the initial guesses 
of the kinetic parameters. In the case of hydrolysis lignin, three constituents are considered, which means that the low proportion of extractives can be joined by half to that of hemicellulose and cellulose. The number of constituents and their proportions which will be considered for each biomass in the present study are summarized in Table 4.

\section{Table 4}

Mass fractions of the four constituents for the three biomass, as deduced from a comparison between Table 3 and the mass rate curves presented in Figure 1.

\begin{tabular}{lrrrr}
\hline & Extractives & Hemicellulose & Cellulose & Lignin \\
\hline T-shirt & 0.0 & 0.0 & 100.0 & 0.0 \\
Palm fiber & 20.0 & 24.0 & 35.0 & 21.0 \\
Hydrol. Lignin & 0.0 & 8.4 & 25.3 & 66.3 \\
\hline
\end{tabular}

Only Mampel, or first-order, reaction function $(f(\alpha)=1-\alpha)$ is here considered for the simulation of the thermal degradation of the three materials. As indicated in section 2.2, initial guesses of the kinetic parameters and an objective function have to be chosen to determine the optimal values of these kinetic parameters. The initial guesses are chosen pair by pair, trying to superimpose the experimental and simulated mass and mass rate curves. Once the initial guesses $(A, E a)$ of the kinetic parameters have been determined, different objective functions have been tested plotting the values of the difference between the experimental and simulated mass and mass rate curves for values of the kinetic parameters lying in the domain $[0.8 \times A, 1.2 \times A] \times[0.8 \times E a, 1.2 \times E a]$. The objective function defined through:

$$
\text { error }=\sum_{j=1}^{J}\left(\left(\frac{d m}{d t}\right)_{\exp }\left(t_{j}\right)\left(\left(\frac{d m}{d t}\right)_{\exp }\left(t_{j}\right)-\left(\frac{d m}{d t}\right)_{s i m}\left(t_{j}\right)\right)^{2}\right)^{2}
$$

seems to present properties which lead to a unique set of optimal values of the kinetic parameters. Here the $t_{j}$ are selected experimental measure times, regularly distributed along the overall experiment duration $t_{\max }$. Taking $J$ approximately equal to 150 indeed reduces in a significant way the computing time. 


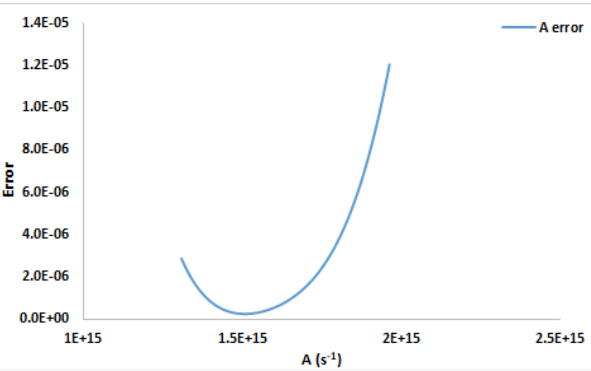

a)

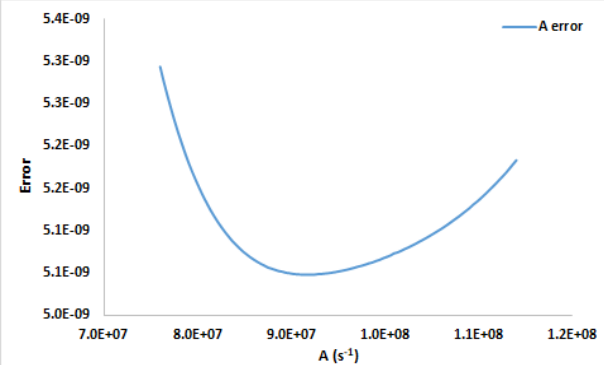

c)

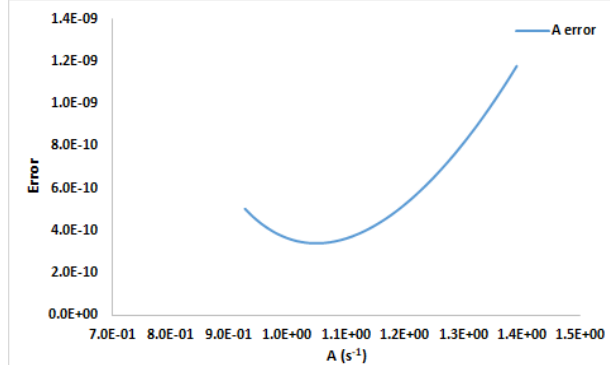

e)

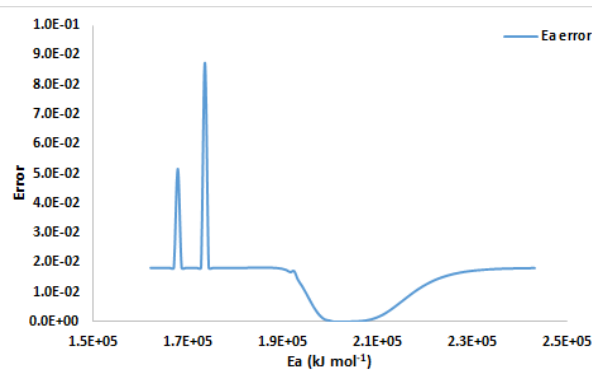

b)

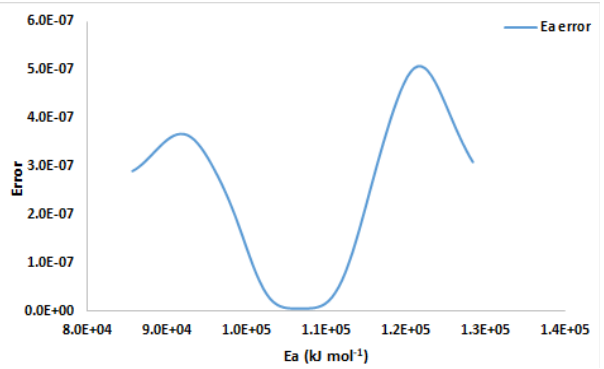

d)

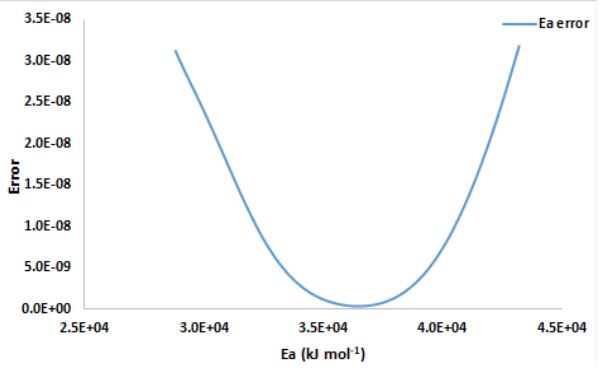

f)

Fig. 2. Values of the objective function with respect to $A$ and $E a$ for T-shirt $(a)$ and $b)$ ), palm fiber $(c)$ and $d)$ ) and hydrolysis lignin $(e)$ and $f)$ ), under nitrogen and temperature ramp of $10{ }^{\circ} \mathrm{C} / \mathrm{min}$. 
Considering variations around the other pairs of kinetic parameters leads to quite similar curves which means that this quartic objective function seems to be adapted to the determination of the optimal kinetic parameters for the pyrolysis of these materials.

The optimal values of the kinetic parameters associated to the thermal degradation of the three materials under nitrogen and a temperature ramp of $10{ }^{\circ} \mathrm{C} / \mathrm{min}$ and simulated through the EIPR model with the first-order reaction function and the objective function given in (4) are gathered in Table 5 , together with the maximal difference between the experimental and simulated mass rate curves.

\section{Table 5}

Optimal values of the kinetic parameters for the thermal degradation of the three materials through the EIPR model under nitrogen and a temperature ramp of $10{ }^{\circ} \mathrm{C} / \mathrm{min}$ and maximal differences between the experimental and simulated mass rate curves.

\begin{tabular}{lrrr}
\hline & T-shirt & Palm fiber & Hydrol. Lignin \\
\hline$A_{1}(1 / \mathrm{s})$ & - & $6.55 \times 10^{7}$ & - \\
$E a_{1}(\mathrm{~kJ} / \mathrm{mol})$ & - & $9.15 \times 10^{4}$ & - \\
$A_{2}(1 / \mathrm{s})$ & - & $1.02 \times 10^{8}$ & $1.30 \times 10^{6}$ \\
$E a_{2}(\mathrm{~kJ} / \mathrm{mol})$ & - & $1.08 \times 10^{5}$ & $9.45 \times 10^{4}$ \\
$A_{3}(1 / \mathrm{s})$ & $1.65 \times 10^{15}$ & $5.92 \times 10^{8}$ & $1.08 \times 10^{9}$ \\
$E a_{3}(\mathrm{~kJ} / \mathrm{mol})$ & $2.03 \times 10^{5}$ & $1.29 \times 10^{5}$ & $1.33 \times 10^{5}$ \\
$A_{4}(1 / \mathrm{s})$ & - & 22.00 & 1.16 \\
$E a_{4}(\mathrm{~kJ} / \mathrm{mol})$ & - & $5.20 \times 10^{4}$ & $3.60 \times 10^{4}$ \\
max. diff. $(\% / \mathrm{s})$ & $3.66 \times 10^{-2}$ & $2.06 \times 10^{-2}$ & $1.18 \times 10^{-2}$ \\
\hline
\end{tabular}

The maximal differences between the experimental and simulated mass rate curves obtained through the EIPR model with the first-order reaction function have to be compared to the maximal height of the experimental mass rate curves. For each material, these maximal differences represent around $1 / 10$ of the maximal height.

The simulated mass and mass rate curves obtained for the three materials are gathered in Figure 3. For each material, the initial mass sample has been normalized at $100 \%$ at $150{ }^{\circ} \mathrm{C}$, for comparison. 


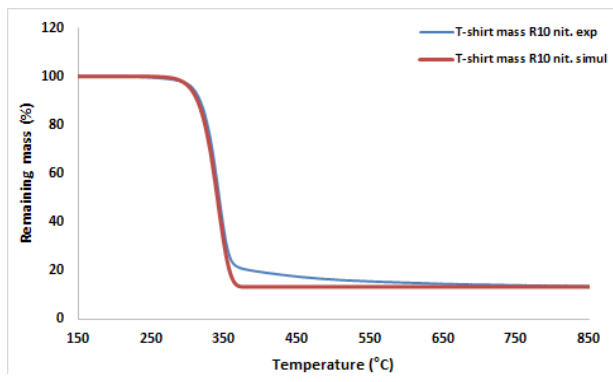

a)

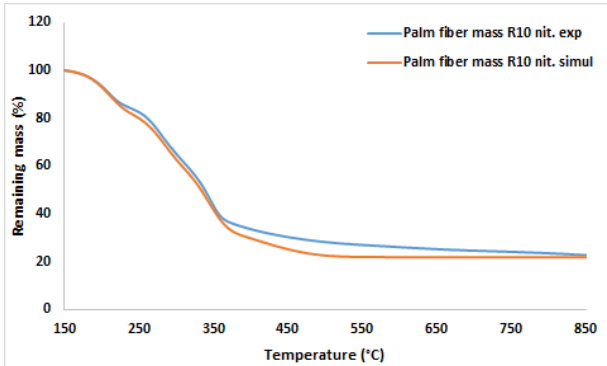

c)

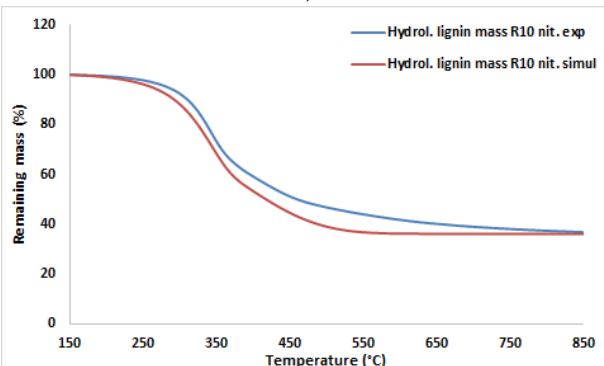

e)

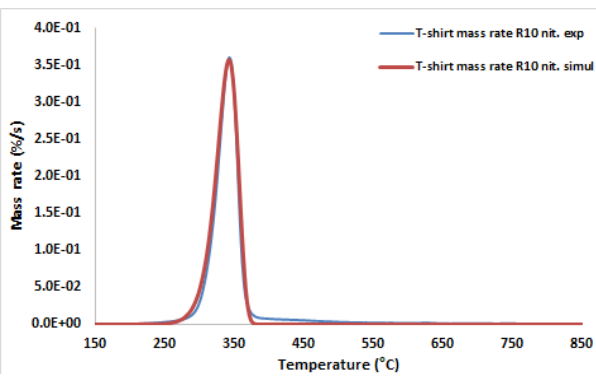

b)

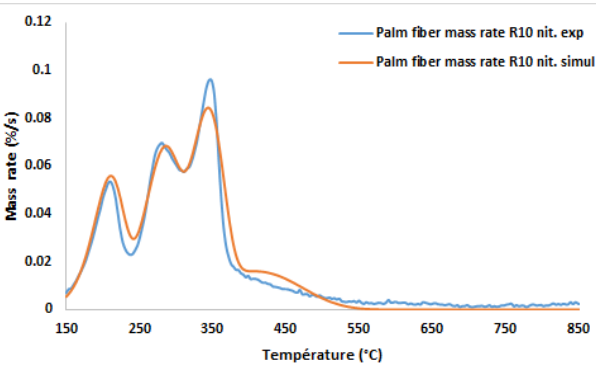

d)

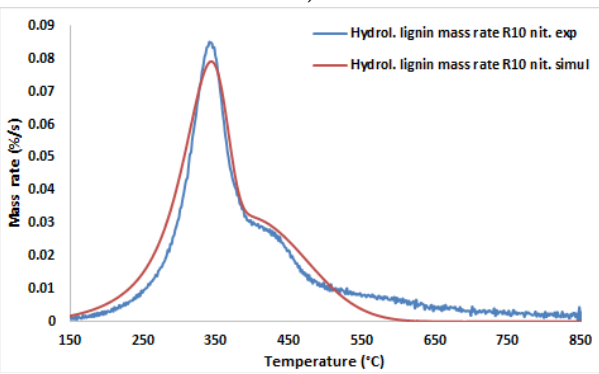

f)

Fig. 3. Experimental (blue) and simulated (red) mass and mass rate curves for T-shirt $(a)$ and $b)$ ), palm fiber $(c)$ and $d)$ ) and hydrolysis lignin $(e)$ and $f)$ ) under nitrogen and a temperature ramp of $10^{\circ} \mathrm{C} / \mathrm{min}$.

Even if the EIPR model is here based on a first-order reaction function, the simulations presented in Figure 3 reflect in a satisfying way the thermal degradation of the materials under nitrogen and for a temperature ramp of $10{ }^{\circ} \mathrm{C} / \mathrm{min}$.

Remark 1. Observing carefully the mass rate curve of hydrolysis lignin, Fig. $1 \mathrm{f})$, it is possible to observe a shoulder and a long tail on the right-hand 
side of the unique devolatilization peak. This may be the consequence of different stages in the pyrolysis of the lignin part of this material which represents around 2/3 of its mass. The pyrolysis of lignin has been analyzed by the different authors who observed different stages corresponding to the degradation of chemical bonds in different temperature ranges, see [27] for example. As a consequence, it is possible to simulate the pyrolysis of hydrolysis lignin considering four constituents: hemicellulose, cellulose and two lignin parts. Taking the proportions of these four constituents respectively equal to 8.4, 25.3, 42.0 and 24.3\%, compare to Table 4, the optimal values of the kinetic parameters returned by the improved EIPR model are: $A_{H}=6.80 \times 10^{6} 1 / \mathrm{s}, E a_{H}=1.05 \times 10^{5} \mathrm{~kJ} / \mathrm{mol}, A_{C}=6.40 \times 10^{8} \mathrm{1} / \mathrm{s}$, $E a_{C}=1.30 \times 10^{5} \mathrm{~kJ} / \mathrm{mol}, A_{L_{1}}=15.21 / \mathrm{s}, E a_{L_{1}}=4.80 \times 10^{4} \mathrm{~kJ} / \mathrm{mol}$ and $A_{L_{2}}=0.681 / \mathrm{s}, E a_{L_{2}}=4.10 \times 10^{4} \mathrm{~kJ} / \mathrm{mol}$. The maximal difference between the experimental and simulated mass rate curves is here equal to $7.8 \times 10^{-3} \% / s$, which is lower than that $\left(1.18 \times 10^{-2} \% / s\right)$ obtained when considering three constituents in the hydrolysis lignin. This proves the importance of the determination of the number of constituents to be considered in a lignocellulosic material and of its proportions.

\subsection{Other methods or models}

The use of an EIPR model, and especially the independence of the thermal degradation of the different constituents of the biomass, is questionable when simulating the pyrolysis of materials, see [29], [30], where the interactions between the constituents of a lignocellulosic material have been analyzed.

Different other methods or models have already been proposed in the literature to simulate the pyrolysis of materials under low temperature ramps.

\subsubsection{The Differential Isoconversional method}

The differential isoconversional method starts with the equation, [7]:

$$
\frac{d \alpha}{d t}(t)=k(T(t)) f(\alpha(t)),
$$

which expresses the variations versus time of the extent of conversion $\alpha$ defined in (2) for the whole material. In the above equation $(5), k(T)$ is given an Arrhenius expression $k(T)=A \exp (-E a /(R T))$ and the function $f$ may take different expressions, see section 2.2. Here $A$ is the pre-exponential factor and $E a$ is the activation energy associated to the thermal degradation 
process of the whole material. Dividing the equation (5) by $f(\alpha(t))$ and taking the logarithm leads to:

$$
\ln \left(\frac{1}{f(\alpha(t))} \frac{d \alpha}{d t}(t)\right)=\ln (k(T(t)))=\ln (A)-\frac{E a}{R T(t)} .
$$

To determine the values of the kinetic parameters $A$ and $E a$, the values of the left-hand side of the preceding equality are plotted for successive values of the extent of conversion and for different temperature ramps (at least three), in terms of $1 / T$. The ICTAC recommendations, [7], suggest to take values of the extent of conversion with steps not larger than 0.05. The parameters of the straight lines which are plotted for different values of the extent of conversion using a linear regression lead to the determination of $\ln (A)$ (hence of $A$ ) and of $E a$. The differential isoconversional method leads to values of the kinetic parameters $A$ and $E a$ which are global for the material but which depend on the extent of conversion.

In the present context, the differential isoconversional method has been applied to simulate the pyrolysis of palm fibers. Three temperature ramps have been considered, namely 5, 10 and $20{ }^{\circ} \mathrm{C} / \mathrm{min}$. Mampel's reaction function has been tested. The linear regressions associated to the equation (6) for values of the extent of conversion between 0.25 and 0.50 lead to the following straight lines represented in Figure 4.

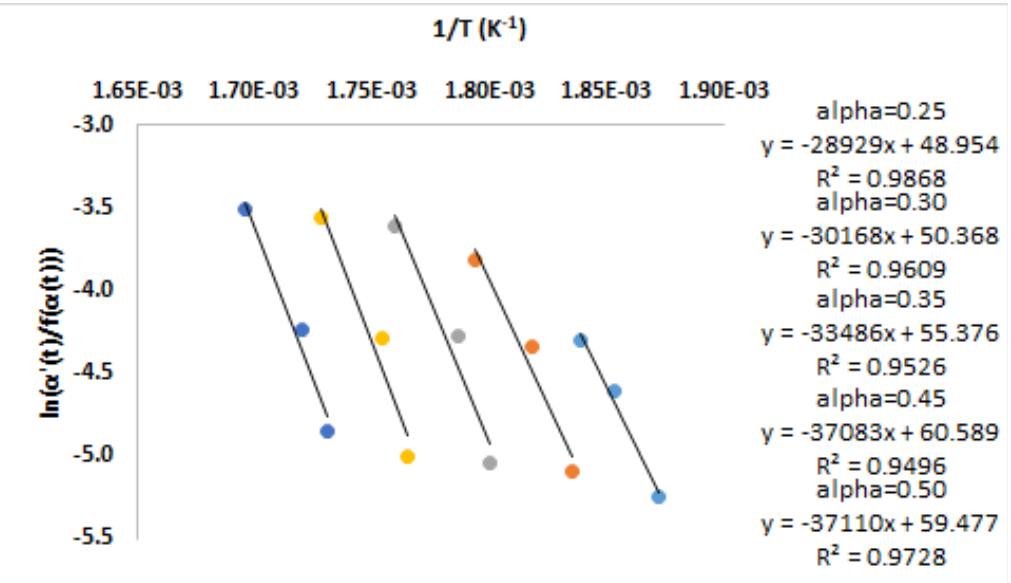

Fig. 4. Linear regressions obtained when applying the differential isoconversional method to the pyrolysis of palm fibers. 
The coefficients of the regression lines lead to the values of the kinetic parameters gathered in Table 6 . The determination coefficients are approximately equal to 0.82 which is low.

\section{Table 6}

Values of the kinetic parameters determined through the differential isoconversional method for the pyrolysis of palm fibers and for successive values of the extent of conversion.

\begin{tabular}{ccrccr}
\hline$\alpha$ & $A(1 / \mathrm{s})$ & $E a(\mathrm{~kJ} / \mathrm{mol})$ & $\alpha$ & $A(1 / \mathrm{s})$ & $E a(\mathrm{~kJ} / \mathrm{mol})$ \\
\hline 0.05 & $1.04 \times 10^{9}$ & 99.9 & 0.55 & $2.51 \times 10^{21}$ & 263.1 \\
0.10 & $1.36 \times 10^{9}$ & 102.4 & 0.60 & $5.87 \times 10^{16}$ & 212.6 \\
0.15 & $2.61 \times 10^{11}$ & 125.3 & 0.65 & $2.05 \times 10^{16}$ & 208.7 \\
0.20 & $9.94 \times 10^{28}$ & 298.7 & 0.70 & $1.48 \times 10^{17}$ & 220.1 \\
0.25 & $1.74 \times 10^{41}$ & 434.7 & 0.75 & $5.49 \times 10^{19}$ & 253.1 \\
0.30 & $1.82 \times 10^{21}$ & 240.5 & 0.80 & $5.21 \times 10^{25}$ & 332.1 \\
0.35 & $7.49 \times 10^{21}$ & 250.8 & 0.85 & $1.46 \times 10^{18}$ & 254.9 \\
0.40 & $1.12 \times 10^{24}$ & 278.4 & 0.90 & $2.67 \times 10^{6}$ & 115.1 \\
0.45 & $2.06 \times 10^{25}$ & 308.3 & 0.95 & $9.01 \times 10^{7}$ & 167.4 \\
0.50 & $6.77 \times 10^{25}$ & 308.5 & & & \\
\hline
\end{tabular}

The values present large variations, especially for low and large values of the extent of conversion, that is when small amounts of materials are being degraded at the beginning or at the end of the pyrolysis process. The mean value of the activation energies for values of the extent of conversion between 0.30 and 0.75 is equal to $254.4 \mathrm{~kJ} / \mathrm{mol}$ and that of the pre-exponential factor is equal to $2.75 \times 10^{25} 1 / \mathrm{s}$. These mean values are much higher than that returned by the EIPR model as indicated in Table 5 .

With these values of the kinetic parameters, it is possible to simulate the thermal degradation under nitrogen of the palm fibers for a temperature ramp equal to $10{ }^{\circ} \mathrm{C} / \mathrm{min}$. First stepwise functions with respect to time $A(t)$ and $E a(t)$ are defined linking the values of the extent of conversion to the corresponding experimental times. Then the equation (5) is solved using Scilab, with the initial value $\alpha(0)=0$. The results of these simulations are gathered in Figure 5. 


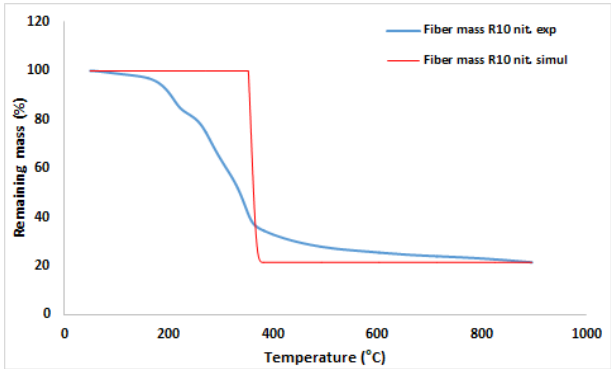

a)

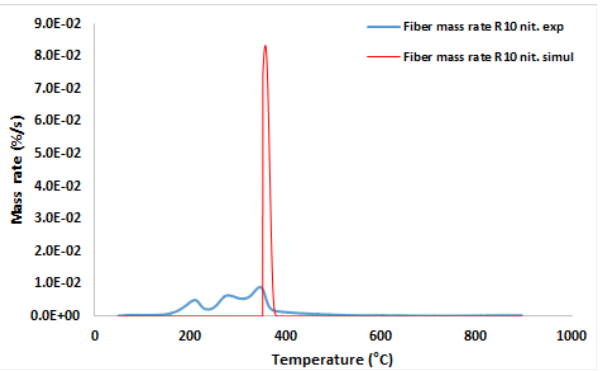

b)

Fig. 5. Experimental (red) and simulated (grey) mass $(a)$ ) and mass rate

(b) ) curves, as returned by the differential isoconversional method considering the pyrolysis of palm fibers under a temperature ramp of 10 ${ }^{\circ} \mathrm{C} / \mathrm{min}$.

In the simulations, the mass starts decreasing later than in the experimental curve but decreases very fast, because of the high values of the kinetic parameters returned by the differential isoconversional method. A unique devolatilization peak appears in the simulated mass rate curve later than in the experimental one, which is too high and too thin.

Such a poor reconstruction process of the thermal degradation of lignocellulosic materials through the differential isoconversional method has already been observed in [28] in the case of cotton residue. One mathematical reason may be the use of logarithm and exponential functions in the construction of the differential isoconversional method, see (6), which may lead to uncontrolled errors. Another mathematical reason may be the use of the first-order reaction in the present case. In the above-described case of cotton residue, a $3 \mathrm{D}$ reaction function (3) has also been tested, but with poor simulations. One chemical reason to this poor agreement may be that the thermal degradation of a lignocellulosic material is not so easy to simulate with the "global" differential isoconversional method.

\subsubsection{The Distributed Activation Energy Method}

A Distributed Activation Energy Method (DAEM) has been used by many authors to simulate the pyrolysis of different lignocellulosic materials. It starts with the ordinary differential equation for the $i$ th constituent of the material: 


$$
\frac{d \alpha_{i}}{d t}(t)=k_{i}(T(t))\left(1-\alpha_{i}(t)\right)^{n_{i}}=A_{i} \exp \left(-\frac{E a_{i}}{R T(t)}\right)\left(1-\alpha_{i}(t)\right)^{n_{i}},
$$

where $\alpha_{i}(t)$ is the extent of conversion of the $i$ th constituent of the material at time $t$ and $n_{i} \geq 1$ is a reaction order. If $n_{i}>1$, the direct integration of this equation (7) leads to:

$$
\alpha_{i}(t)=1-\left(1+(n-1) \int_{0}^{t} A_{i} \exp \left(-\frac{E a_{i}}{R T(s)}\right) d s\right)^{1 /(1-n)},
$$

hence to

$$
\frac{d \alpha_{i}}{d t}(t)=\left(1+(n-1) \int_{0}^{t} A_{i} \exp \left(-\frac{E a_{i}}{R T}\right) d s\right)^{n /(1-n)} A_{i} \exp \left(-\frac{E a_{i}}{R T(t)}\right)
$$

If $n_{i}=1$, the direct integration of the equation (7) leads to:

$$
\alpha_{i}(t)=1-\exp \left(-\int_{0}^{t} A_{i} \exp \left(-\frac{E a_{i}}{R T(s)}\right) d s\right)
$$

hence to

$$
\frac{d \alpha_{i}}{d t}(t)=\exp \left(-\int_{0}^{t} A_{i} \exp \left(-\frac{E a_{i}}{R T}\right) d s\right) A_{i} \exp \left(-\frac{E a_{i}}{R T(t)}\right) .
$$

The DAEM method consists to replace the activation energy $E a_{i}$ of the $i$ th constituent by a continuous distribution of activation energies with density $f(E)$. Formula (8) becomes:

$$
\begin{aligned}
& \frac{d \alpha_{i}}{d t}(t)=\int_{0}^{\infty}\left(1+(n-1) \int_{0}^{t} A_{i} \exp \left(-\frac{E}{R T(s)}\right) d s\right)^{n /(1-n)} \\
& \quad \times A_{i} \exp \left(-\frac{E}{R T(t)}\right) f(E) d E
\end{aligned}
$$

and formula (9) becomes:

$$
\begin{aligned}
& \frac{d \alpha_{i}}{d t}(t)=\int_{0}^{\infty} \exp \left(-\int_{0}^{t} A_{i} \exp \left(-\frac{E}{R T(s)}\right) d s\right) \\
& \quad \times A_{i} \exp \left(-\frac{E}{R T(t)}\right) f(E) d E .
\end{aligned}
$$


The density $f(E)$ may be taken as a Gaussian, or a Weibull, or a logistic function. It depends on a mean value $E_{0, i}$ and on a standard deviation $\sigma_{i}$, both given in $\mathrm{kJ} / \mathrm{mol}$.

Assuming that the degradations of the constituents of the material occur through independent parallel reactions and that the fractions $c_{i}$ of these constituents are known, the overall derivative of the extent of conversion with respect to time is given as:

$$
\frac{d \alpha}{d t}(t)=\sum_{i=1}^{I} c_{i} \frac{d \alpha_{i}}{d t}(t)
$$

where $c_{i}$ is the proportion of the $i$ th constituent of the material, $i=1, \ldots, I$. The triples $\left(n_{i}, E_{0, i}, \sigma_{i}\right)$ are determined through an optimization procedure which consists to minimize the least-square function or equivalent objective functions:

$$
\sum_{j=1}^{J}\left(\left(\frac{d \alpha}{d t}\right)_{\exp }\left(t_{j}\right)-\left(\frac{d \alpha}{d t}\right)_{s i m}\left(t_{j}\right)\right)^{2} .
$$

In the present study, the pyrolysis of T-shirt and of palm fibers under a temperature ramp of $10^{\circ} \mathrm{C} / \mathrm{min}$ has been simulated using the DAEM method with the Gaussian density $f(E)=\frac{1}{\sigma_{i} \sqrt{2 \pi}} \exp \left(-\frac{\left(E-E_{0, i}\right)^{2}}{2 \sigma_{i}^{2}}\right)$ and taking the reaction orders $n_{i}, i=1$ or $i=1, \ldots, 4$ equal to 1 . The integral occurring in (11) has been reduced to that on $\left[E_{0, i}-5 \sigma_{i}, E_{0, i}+5 \sigma_{i}\right]$ and has been calculated with the Gauss-Lobatto algorithm with five points, dividing this interval into 200 subintervals. The optimization step of the DAEM method has not been considered in the present simulations and the values of the kinetic parameters have been taken from Table 5 , the mean values $E_{0, i}$ of the activation energies being taken equal to $E a_{i}$. In the case of T-shirt, the standard deviations $\sigma$ has been taken equal to $8.0 \mathrm{~kJ} / \mathrm{mol}$. In the case of palm fibers, the standard deviations $\sigma_{i}$ have been taken equal to $1.0 \mathrm{~kJ} / \mathrm{mol}$. The simulations seem to be relatively insensitive with respect to these standard deviations.

The results of the simulations under these hypotheses are gathered in Figure 6. 


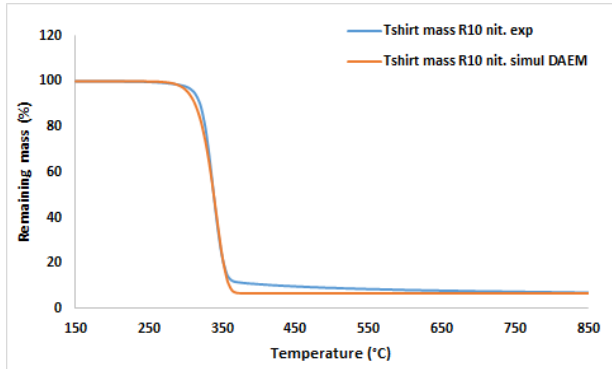

a)

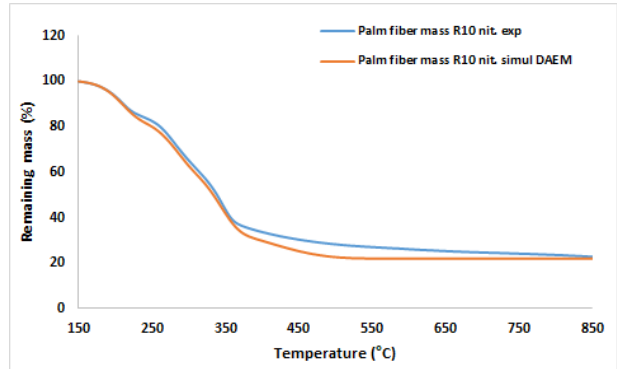

c)

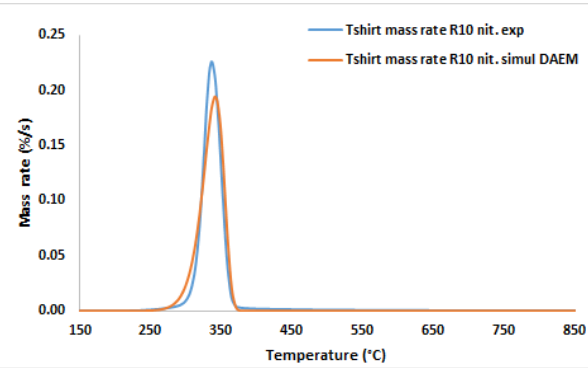

b)

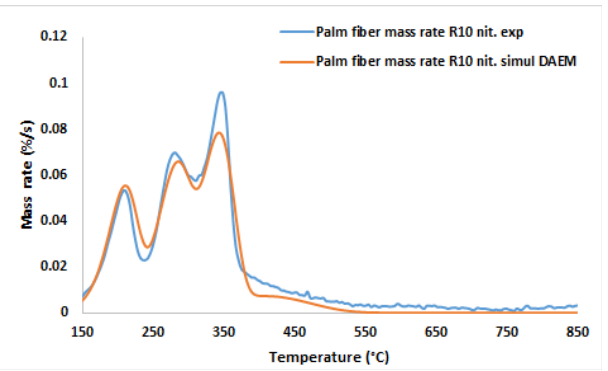

d)

Fig. 6. Experimental (blue) and simulated (red) mass and mass rate curves, as returned by the DAEM model considering the pyrolysis of Tshirt $(a))$ and $(b))$ or palm fibers $(c))$ and $(d))$ under a temperature ramp of $10{ }^{\circ} \mathrm{C} / \mathrm{min}$.

These simulated curves are in good agreement with the experimental ones. In the case of T-shirt, the maximal difference between the experimental and simulated mass rate curves is equal to $4.86 \times 10^{-2} \% / \mathrm{s}$, to be compared to the $3.66 \times 10^{-2} \% / \mathrm{s}$ obtained with the EIPR model. In the case of palm fibers, the maximal difference between the experimental and simulated mass rate curves is equal to $1.92 \times 10^{-2} \% / \mathrm{s}$, to be compared to the $2.06 \times 10^{-2} \% / \mathrm{s}$ obtained with the EIPR model.

In [31], the authors applied the DAEM method to simulate the pyrolysis of lignocellulosic materials using the logistic function and a reaction order $n=$ 2.0 and they found a pre-exponential factor equal to $10^{10} 1 / \mathrm{s}$, an activation energy equal to $150 \mathrm{~kJ} / \mathrm{mol}$ a standard deviation $\sigma=10 \mathrm{~kJ} / \mathrm{mol}$.

The advantages and disadvantages of the three methods or models which have been presented may be summarized as:

- The three method and models are based on similar first-order differential equations which express the evolution with respect to time either 
of the global extent of conversion (in the case of the DIC method) or of the mass or extent of conversion of each constituent of the lignocellulosic material (in the cases of EIPR or DAEM models). The DIC method considers only one differential equation. The EIPR model considers as many differential equations as the number of constituents to be considered. This number is derived from the characterization of the material through Van Soest's protocol and from the observation of the experimental mass rate curve. The DAEM model considers an infinite series of differential equations.

- The EIPR and DAEM models return a unique couple of kinetic parameters (pre-exponential factor and activation energy) for each constituent of the lignocellulosic material. These kinetic parameters are independent of the time parameter. On the contrary, the DIC method returns a unique couple of kinetic parameters which are global for the material. But this couple depends on the extent of conversion, hence on the time parameter.

- The EIPR and DAEM models are based on the hypothesis that the thermal degradations of the constituents of a lignocellulosic material occur in an independent way. These models need the proportions of the constituents of the lignocellulosic material. As previously described, these proportions may be obtained applying Van Soest's protocol. But these experimental proportions may sometimes be corrected, because the thermal degradation of the material involves complex chemical reactions.

- The EIPR model needs the resolution of first-order differential equations describing the evolutions with respect to time of the remaining mass of each constituent of the sample or of the associated extent of conversion. The DAEM model converts this differential equation through an integration "by hand" to an expression of the extent of conversion in terms of an integral, whose computation needs a software. The DAEM model further introduces a continuous density of activation energies through a second integral with respect to the activation energies. Both models require a software to determine the optimal values of the kinetic parameters. The DIC model only requires a software which computes linear regressions for successive sets of at least three points (experimental thermogravimetric results corresponding to at least three different 
temperature ramps).

- The EIPR and DAEM models lead to almost the same optimal values of the kinetic parameters. They return simulations of the mass and mass rate curves which are in a good agreement with the experimental ones. On the contrary, the reconstruction process of these mass and mass rate curves based on the values of the kinetic parameters returned by the DIC model are not in good agreement with the experimental curves.

\section{Conclusion}

Throughout the present study, the thermal degradation of three different biomass has been analyzed under a non-oxidative atmosphere and under the same temperature ramp of $10{ }^{\circ} \mathrm{C} / \mathrm{min}$. These three biomass have first been characterized and especially their mass fractions of extractives, hemicellulose, cellulose and lignin have been determined through Van Soest's protocol. To simulate the thermal degradation of these biomass, the EIPR model has first been used with a first-order reaction function. This leads to first-order differential equations which express the evolution of the mass of each constituent of the biomass with respect to time. The number of constituents to be considered and their proportions have been deduced from the results returned by Van Soest's protocol and observing the experimental mass rate curves. The ordinary differential equations have been solved using Scilab software. The optimal values of the kinetic parameters have been determined building a quartic objective function to be minimized, which involves the experimental mass rate. This objective function leads to a unique couple of kinetic parameters for each constituent of the biomass. For each biomass, the maximal difference between the experimental and simulated mass rate curves has been computed which proves that the simulated mass and mass rate curves are in good agreement with the experimental ones.

The thermal degradations under nitrogen of these biomass have also been simulated with the differential isoconversional method and with a Distributed Activation Energy Model. The advantages and disadvantages of these model or methods have finally been compared.

Even if the present study focused on the simulation of the pyrolysis of these three materials and under the same temperature ramp of $10{ }^{\circ} \mathrm{C} / \mathrm{min}$, the tools which are proposed can be applied to simulate their pyrolysis under other low temperature ramps. The same tools can also be applied to simulate 
the pyrolysis of other lignocellulosic materials. Finally, the same tools can be applied to simulate the combustion of lignocellulosic materials under low temperature ramps, as the model here involves a further differential equation which describes the evolution of the mass of the char which is burnt.

Acknowledgements We thank Mrs. Damaris Kehrli for her careful realization of the slow pyrolysis experiments which have been performed in preparation of the present study.

\section{References}

[1] B. Biswas, R. Singh, J. Kumar, R. Singh, P. Gupta, B.B. Krishna, T. Bhaskar, Pyrolysis behavior of rice straw under carbon dioxide for production of bio-oil. Renew. Energy 129 (2018) 686-694. http://dx.doi.org/10.1016/j.renene.2017.04.048

[2] V. Dhyani, T. Bhaskar, A comprehensive review on the pyrolysis of lignocellulosic biomass, Renew. Energy 129 (2018) 695-716. https://doi.org/10.1016/j.renene.2017.04.035

[3] B. Babu, Biomass pyrolysis: a state-of-the-art review, Biofuels Bioprod. Biorefining 2 (2008) 393-414. https://doi.org/10.1002/bbb.92

[4] F.X. Collard, J. Blin, A review on pyrolysis of biomass constituents: Mechanisms and composition of the products obtained from the conversion of cellulose, hemicelluloses and lignin, Renew. Sustain. Energy Rev. 38 (2014) 594-608. http://dx.doi.org/10.1016/j.rser.2014.06.013

[5] G. Dorez, L. Ferry, R. Sonnier, A. Taguet, J.M. Lopez-Cuesta, Effect of cellulose, hemicellulose and lignin contents on pyrolysis and combustion of natural fibers, J. Anal. Appl. Pyrol. 107 (2014) 323-331. http://dx.doi.org/10.1016/j.jaap.2014.03.017

[6] D. Vamvuka, E. Kakaras, E. Kastanaki, P. Grammelis, Pyrolysis characteristics and kinetics of biomass residuals mixtures with lignite, Fuel 82 (2003) 1949-1960. http://dx.doi.org/10.1016/S0016-2361(03)00153-4

[7] S. Vyazovkin, A.K. Burnham, J.M. Criado, L.A. PerezMaqueda, C. Popescu, N. Sbirrazuoli ICTAC Kinetics Committee recommendations for performing kinetic computations 
on thermal analysis data, Thermochim. Acta 520 (2011) 1-19. http://dx.doi.org/10.1016/j.tca.2011.03.034

[8] T. Damartzis, D. Vamvuka, S. Sfakiotakis, A. Zabaniotou, Thermal degradation studies and kinetic modeling of cardoon (Cynara cardunculus) pyrolysis using thermogravimetric analysis (TGA), Bioresour. Technol. 102 (2011) 6230-6238. http://dx.doi.org/10.1016/j.biortech.2011.02.060

[9] Y. Liang, B. Cheng, Y. Si, D. Cao, H. Jiang, G. Han, X. Liu, Thermal decomposition kinetics and characteristics of Spartina alterniflora via thermogravimetric analysis, Renew. Energy 68 (2014) 111-117. http://dx.doi.org/10.1016/j.renene.2014.01.041

[10] B. Fidalgo, M. Chilmeran, T. Somorin, A. Sowale, A. Kolios*, A. Parker, L. Williams, M. Collins, E.J. McAdam, S. Tyrrel, Nonisothermal thermogravimetric kinetic analysis of the thermochemical conversion of human faeces, Renew. Energy 132 (2019) 1177-1184. https://doi.org/10.1016/j.renene.2018.08.090

[11] K. Papadikis, S. Gub, A.V. Bridgwater, H. Gerhauser, Application of CFD to model fast pyrolysis of biomass, Fuel Process. Technol. 90 (2009) 504-512. doi:10.1016/j.fuproc.2009.01.010

[12] Q. Xiong, F. Xu, Y. Pan, Y. Yang, Z. Gao, S. Shu, K. Hong, F. Bertrand, J. Chaouki, Major trends and roadblocks in CFD-aided process intensification of biomass pyrolysis, Chem. Eng. Process.: Process Intens. 127 (2018) 206-212. https://doi.org/10.1016/j.cep.2018.04.005

[13] Q. Xiong, J. Zhang, F. Xu, G. Wiggins, C. Stuart Daw, Coupling DAEM and CFD for simulating biomass fast pyrolysis influidized beds, J. Anal. Appl. Pyrol. 117 (2016) 176-181. http://dx.doi.org/10.1016/j.jaap.2015.11.015

[14] H. Zhong, Q. Xiong, Y. Zhu, S. Liang, J. Zhang, B. Niu, X. Zhang, CFD modeling of the effects of particle shrinkage and intra-particle heat conduction on biomass fast pyrolysis, Renew. Energy 141 (2019) 236245. https://doi.org/10.1016/j.renene.2019.04.006

[15] Q. Xiong, M.M. Yeganeh, E. Yaghoubi, A. Asadi, M.H. Doranehgard, K. Hong, Parametric investigation on biomass gasification in a fluidized bed 
gasifier and conceptual design of gasifier, Chem. Eng.Process.: Process Intens. 127 (2018) 271-291. https://doi.org/10.1016/j.cep.2018.04.003

[16] P.J. Van Soest, J.B. Robertson, B.A. Lewis, Methods for dietary fiber, neutral detergent fiber, and nonstarch polysaccharides in relation to animal nutrition, J. Dairy Sci. 74 (1991) 3583-3597, doi:10.3168/jds.S00220302(91)78551-2

[17] X. Zhang, H. Deng, X. Hou, R. Qiu, Z. Chen, Pyrolytic behavior and kinetic of wood sawdust at isothermal and nonisothermal conditions, Renew. Energy 142 (2019) 284-294. https://doi.org/10.1016/j.renene.2019.04.115

[18] M.L. Rabinovich, O. Fedoryak, G. Dobele, A. Andersone, B. Gawdzik, ME. Lindstrom, O. Sevastyanova, Carbon adsorbents from industrial hydrolysis lignin: The USSR/Eastern European experience and its importance for modern biorefineries, Renew. Sustain. Energy Rev. 57 (2016) 1008-1024. http://dx.doi.org/10.1016/j.rser.2015.12.206

[19] S. Wang, G. Dai, H. Yang, Z. Luo, Lignocellulosic biomass pyrolysis mechanism: A state-of-the-art review, Progr. Energy Combust. Sci. 62 (2017) 33-86. http://dx.doi.org/10.1016/j.pecs.2017.05.004

[20] M. Van de Velden, J. Baeyens, A. Brems, B. Janssens, R. Dewil, Fundamentals, kinetics and endothermicity of the biomass pyrolysis reaction, Renew. Energy 35 (2010) 232-242

[21] F. Peng, P. Peng, F. Xu, R.C. Sun, Fractional purification and bioconversion of hemicelluloses, Biotechnol. Adv. 30 (2012) 879-903

[22] D.F. Arseneau, Competitive reactions in the thermal decomposition of cellulose, Canad. J. Chem. 49 (1971) 632-638.

[23] A.M. Emsley, G.C. Stevens, Kinetics and mechanisms of the lowtemperature degradation of cellulose, Cellulose 1 (1994) 26-56. doi:10.1007/BF00818797

[24] H.Z. Ding, Z.D. Wang, On the degradation evolution equations of cellulose, Cellulose 15 (2008) 205-224. doi:10.1007/s10570-007-9166-4 
[25] A. Anca-Couce, R. Scharler, Modelling heat of reaction in biomass pyrolysis with detailed reaction schemes, Fuel 206 (2017) 572-579. http://dx.doi.org/10.1016/j.fuel.2017.06.011

[26] M. Valente, A. Brillard, C. Schönnenbeck, J.F. Brilhac, Investigation of grape marc combustion using thermogravimetric analysis. Kinetic modeling using an extended independent parallel reaction (EIPR), Fuel Process. Technol. 131 (2015) 297-303. http://dx.doi.org/10.1016/j.fuproc.2014.10.034

[27] H. Kawamoto, Lignin pyrolysis reactions, J. Wood Sci. 63 (2017) $117-$ 132. DOI 10.1007/s10086-016-1606-z

[28] A. Brillard, D. Habermacher, J.F. Brilhac, Thermal degradations of used cotton fabrics and of cellulose: kinetic and heat transfer modeling, Cellulose 24 (2017) 1579-1595. DOI 10.1007/s10570-017-1200-6

[29] J. Yu, N. Paterson, J. Blamey, M. Millan, Cellulose, xylan and lignin interactions during pyrolysis of lignocellulosic biomass, Fuel 191 (2017) 140-149. http://dx.doi.org/10.1016/j.fuel.2016.11.057

[30] J. Wang, B. Shen, D. Kang, P. Yuan, C. Wu, Investigate the interactions between biomass constituents during pyrolysis using insitu DRIFTS and TGA, Chem. Eng. Science 195 (2019) 767-776. https://doi.org/10.1016/j.ces.2018.10.023

[31] J. Cai, C. Jin, So. Yang, Y. Chen, Logistic distributed activation energy model - Part 1: Derivation and numerical parametric study, Bioresour. Technol. 102 (2011) 1556-1561. doi:10.1016/j.biortech.2010.08.079 